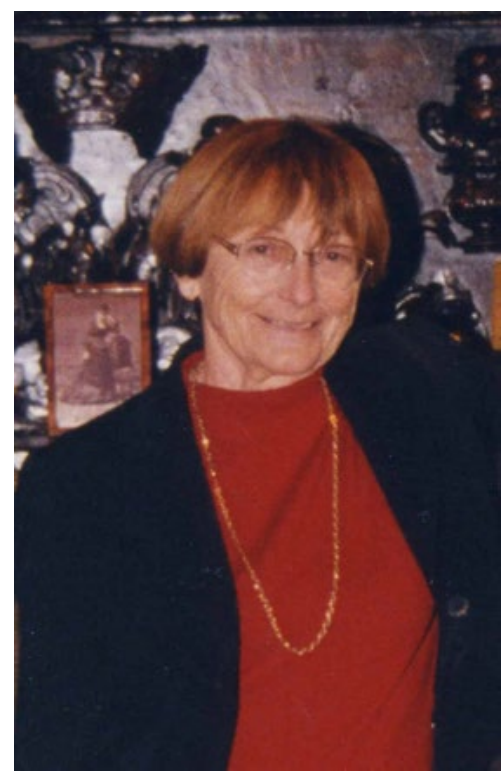

Nelly Clemessy

(1929-2017)

IN MEMORIAM 


\title{
En la desaparición de la maestra y decana del pardobazanismo: Nelly Clemessy (1929-2017)
}

\author{
José Manuel González Herrán \\ (UNIVERSIDADE DE SANTIAGO DE COMPOSTELA) \\ josemanuel.gonzalez.herran@usc.es
}

De nuevo, y por tercera vez desde la fundación de La Tribuna, asumo el penoso deber de anotar otra baja en las huestes del pardobazanismo: en el número 7 (2009) fue Benito Varela Jácome ${ }^{1}$; en el número 11 (2016), Ángeles Quesada Novás²; en este número 12, Nelly Clemesy, fallecida el pasado 28 de septiembre de 2017. Y resulta dolorosamente significativo notar la relación entre esas tres desapariciones: cuando en esta revista me referí a la del primero, recordé que era "no sólo el decano del pardobazanismo, sino principal responsable (junto a Nelly Clemessy: me consta su mutuo aprecio y admiración recíproca) del «descubrimiento» y recuperación que de la obra de doña Emilia se produjo a partir de los años setenta"; por su parte, la hispanista francesa que ahora acaba de dejarnos había presidido el Tribunal que juzgó la tesis doctoral de Quesada Novás. Sin olvidar la antigua vinculación de los tres a nuestra revista, según ha venido constando en sus páginas de créditos: Quesada Novás, como miembro de su Consello de Redacción, a partir del número 2; Varela Jácome y Clemessy, de su Comité Científico, desde el primer número.

Fue precisamente en esa entrega inicial de esta revista cuando Cristina Patiño Eirín y quien esto firma, cumpliendo el encargo -tan grato como honroso- de su Director, mantuvimos con aquella maestra una "Entrevista a Nelly Clemessy (con Emilia Pardo Bazán al fondo $)^{\prime \prime 3}$, cuya relectura es ahora tan pertinente como emotiva; especialmente en sus párrafos iniciales, donde nuestra colega y amiga evocaba los hitos fundamentales de su vocación y dilatada dedicación pardobazanista, a partir de su primera visita a "Marineda":

En 1953, La Coruña me cautivó en el acto. Me gustó su alegre animación y fui descubriendo con verdadero placer la belleza de sus Cantones y de la Ciudad Vieja, tan recoleta y poética. En aquel entonces, era una lindísima ciudad provinciana, fiel a sus costumbres tradicionales que a mí me recordaban los ambientes algo anticuados de mi niñez en Bretaña. En mi reciente visita he descubierto una urbe extensa y moderna pero, afortunadamente, lo antiguo queda preservado, a menudo renovado y embellecido. $\mathrm{Ha}$

\footnotetext{
$\overline{1}$ J. M. G. H., "Recuerdo de un maestro: Benito Varela Jácome (1919-2010)”, La Tribuna. Cadernos de Estudios da Casa Museo Emilia Pardo Bazán, 7 (2009), pp. 23-31

2 J. M. G. H., "Semblanza y recuerdo de una pardobazanista”, La Tribuna. Cadernos de Estudios da Casa Museo Emilia Pardo Bazán, 11 (2016), pp. 11-16

${ }^{3}$ La Tribuna. Cadernos de Estudios da Casa Museo Emilia Pardo Bazán, 1 (2003), pp. 203-213.
} 
perdido el centro desde el atardecer su vida callejera, en cambio la ciudad ha ganado mucho del lado del mar con su magnífico paseo hacia la Torre de Hércules.

Una visita que, lógicamente, se debía a su interés por la obra de nuestra autora, que explicaba así:

Lo que me atrajo al principio en Emilia Pardo Bazán fue su narrativa de ambiente gallego, pero al adentrarme algo más en el estudio no tardé en admirarme de la amplitud, variedad y vigor de su talento de polígrafa. Acababa de descubrir una figura femenina excepcional en la España de su tiempo. Quedé como hechizada y con afán de saber siempre más de ella.

Pasaba luego revista al estado de la cuestión en los estudios pardobazanianos y sus avances de los cincuenta años transcurridos desde que ella lo abordó; la necesidad de una "biografía rigurosa y totalmente fiable"; los aspectos aún pendientes de estudio, especialmente en su teatro y en su poesía; la recuperación de inéditos que por entonces -hace casi quince años- estábamos iniciando (y que no contaba con su aprobación: "me parece excluido emprender la edición en librería de este tipo de textos", declaraba); su propia tarea como traductora (de Los Pazos de Ulloa al francés); su reivindicación del feminismo pardobazaniano: "pareció osado en la España de su tiempo. En realidad era moderado y nada revolucionario. Adoptó posturas realistas al centrar sus reivindicaciones sobre unos problemas claves: la educación e instrucción de la mujer y su posible independencia económica (...) hoy en día su feminismo puede parecer arcaico; pero, personalmente, opino que, en lo esencial, sus ideas siguen vigentes". Y, con una modestia admirable, formulaba en estos términos la consideración que le merecían sus propias aportaciones:

Estoy convencida de que todavía queda mucho que decir sobre las novelas y más todavía sobre los cuentos de Pardo Bazán. Lo mío ha sido una aproximación global, incompleta, como ocurre cuando se abarca una obra tan vasta y polifacética. El mundo de ficción creado por la novelista se presta todavía a múltiples estudios. He abierto un camino, nada más: me doy por muy contenta si he contribuido un poco a despertar el interés por la obra de doña Emilia.

Según consta en el curriculum y relación de publicaciones adjuntos, su primera aproximación a la literatura de la escritora coruñesa fue su Memoria de Licenciatura (equivalente a lo que entre nosotros se llamaba tesina), realizada bajo la dirección de Charles Vincent Aubrun -una de la figuras más eminentes del hispanismo francés del siglo XX- y firmada con su nombre de soltera, Nelly Legal: La Comtesse de Pardo Bazán devant les grandes préoccupations de son temps (Paris, 1954); aunque inédita, podemos hacernos una idea aproximada de su contenido, probablemente resumido en la conferencia que Nelly pronunció en A Coruña como parte de un ciclo de lecciones que conmemoraba en 1971 el cincuentenario del fallecimiento de doña Emilia, y que en ese mismo año se publicó en la Revista 'José Cornide' de Estudios Coruñeses: "El ideario de doña Emilia Pardo Bazán: sus ideas estéticas, sus preocupaciones políticas y sociales". 
Para esa fecha, Nelly Legal se había doctorado en 1968 con una tesis, Emilia Pardo Bazán, contes perdus et retrouvés, de más de ochocientas páginas mecanografiadas en cuatro cuadernos: el primero dedicado al estudio introductorio y a los imprescindibles índices y catálogo; los otros tres, a la transcripción de unos 160 relatos breves de nuestra autora, hasta entonces olvidados en la prensa periódica española, y prácticamente desconocidos, pues doña Emilia no los había recogido en ninguno de los quince libros de cuentos que publicó.

Lamentablemente, esa monumental aportación quedó inédita en su parte más valiosa -la transcripción de los cuentos-, aunque su inventario, con detallada indicación de las fechas y las cabeceras en que cada uno de ellos había aparecido, se incluyó en su librito de 1972, firmado ya como Nelly Clemessy [a ese propósito, ella misma nos explicó que el apellido se escribía sin tilde en la primera 'e', y no como muchos veníamos haciendo: Clémessy]: Les Contes d'Emilia Pardo Bazán (Essai de classification), minucioso catálogo de esa parcela de su producción, y que durante mucho tiempo ha sido fuente inexcusable para el estudio de la narrativa breve de la Condesa. Una fuente tan utilizada (y no siempre citada), como frecuentemente saqueada; igual que acaso también lo fueron los cuadernos inéditos de su tesis, según cabe deducir de algo que ella misma nos contó: en sus gestiones para publicar íntegramente aquellos materiales había enviado a una editorial la única copia que conservaba de los cuadernos mecanografiados; pero ni la edición prosperó ni los originales le fueron devueltos. Por sorprendente que pueda parecer, Nelly no volvió a ver aquellas páginas hasta que yo mismo (cuando preparaba los volúmenes XI y XII de las Obras Completas $)^{4}$ pude conseguir una copia de los cuadernos depositados en los archivos de la Université de Montpellier, que fotocopié y envié a su legítima propietaria intelectual. Aquel envío, y también la dedicatoria que puse a mi edición ("A Nelly Clemessy, maestra y amiga, pionera en la recuperación de los cuentos dispersos de Emilia Pardo Bazán"), pretendían compensar de alguna manera el disgusto que supuse le habría producido el cotejo de su tesis con algunos "rescates" de cuentos pardobazanianos, publicados después de 1968: cotejo que hace evidentes coincidencias tan notorias que alcanzan, incluso, a algunas erratas.

\footnotetext{
4 Emilia Pardo Bazán, Obras Completas: vol. XI, Cuentos dispersos I (1865-1910); vol. XII, Cuentos dispersos II (1911-1921), ed. de J. M. González Herrán, Madrid: Biblioteca Castro, 2011
} 


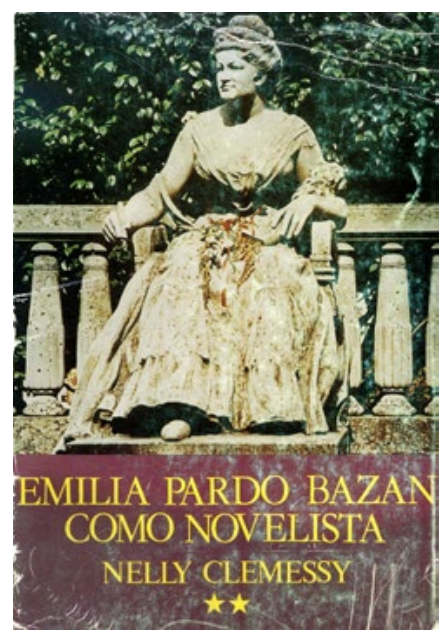

Pero siendo importantísimas las aportaciones que hasta aquí he reseñado, la culminación de su tarea pardobazanista fue la presentación en la Université de la Sorbonne, en 1970, de lo que en Francia se denomina "Thèse d'État", que dos años más tarde recogería en los dos volúmenes de Emilia Pardo Bazán, romancière (La critique, la théorie, la pratique), y traducidos al español en 1981 [aunque con fecha editorial de 1982] como Emilia Pardo Bazán como novelista. De la teoría a la práctica: monumental monografía que se ha convertido en la referencia inexcusable para quien pretenda aproximarse a la literatura -y no solo a la narrativa- de nuestra escritora. A ello cabe añadir sus valiosos artículos, notas, reseñas, ensayos, ponencias y comunicaciones, recogidos en homenajes, volúmenes colectivos, actas de congresos y revistas (como La Tribuna, cuyas páginas se han honrado publicando sus últimos trabajos). Sin olvidar su edición prologada y anotada de Los Pazos de Ulloa (1987), que también tradujo al francés como Le Château d’Ulloa (1990).

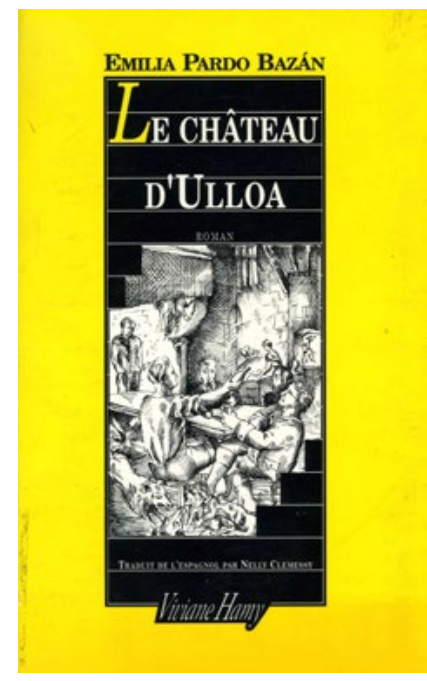


Además de esos trabajos, muchos de los cuales se han convertido en referencias bibliográficas inexcusables, Nelly se ocupó también de otros autores y temas de la historia cultural o literaria española: el trovador Macías O Namorado, las mujeres en la RAE, Domenico Scarlatti, Rosalía de Castro, Alfredo Brañas, Benito Pérez Galdós, Jacinto Octavio Picón, Ramón del Valle-Inclán, Carmen de Burgos...

Junto a su labor investigadora, nuestra recordada maestra desarrolló una dilatada carrera universitaria, iniciada muy pronto en Paris y, ya desde 1961, en Niza, Universidad donde desempeñó, además de su tarea como formadora de varias generaciones de profesores de español, importantes responsabilidades de organización y gestión, hasta que en 1989, por razones personales, obtuvo la jubilación, que fue acompañada de su nombramiento como Profesora Emérita.

Retirada en su casa de "Le Pin Penché", en Valescure, se dedicó preferentemente a cuidar de su esposo -enfermo y de más edad que ella-, sin abandonar sus dos grandes pasiones: la lectura y la investigación. Excepcionalmente hizo algunos viajes: entre ellos, a Coruña y a Santiago de Compostela, para participar en simposios pardobazanianos, investigar en sus archivos y bibliotecas, o presidir Tribunales de tesis doctorales. Su esposo falleció en julio de 2017 y Nelly le siguió cuando apenas habían transcurrido tres meses.

\section{CURRICULUM Y PUBLICACIONES DE NELLY [LEGAL] CLEMESSY 5}

Nacida en Nantes en 1929, hizo sus estudios secundarios en París y los universitarios en la Sorbonne entre 1950-1956, año en el que obtiene la Agrégation d'Espagnol. Al año siguiente su maestro Charles V. Aubrun le encarga de las tareas de Assistante en el Institut d'Études Hispaniques de París. De allí pasa al Lycée Calmette, en Nice y desde 1961 es Assistante en el Collège Littéraire Universitaire de la misma ciudad. Poco después, el Profesor André Joucla Ruau, Director del Département Hispanique et Ibéro-Américain de la Universidad de Aix-en-Provence le encarga que ponga en marcha una sección en Nice, cuya Universidad aún no se ha creado. En 1965, Nelly Clemessy funda el Département Hispanique de la Faculté des Lettres en Nice, que dirigirá hasta 1978.

Se doctora en el curso 1967-68 con la "Thèse de Doctorat de 3ème cycle, présentée et publiquement soutenue devant la Faculté des Lettres et Sciences Humaines à l'Université de Montpellier", Emilia Pardo Bazán, contes perdus et retrouvés, dirigida por el Prof. JeanLouis Fleckniakoska.

En 1970, en la Sorbonne, recibe el grado de Docteur d'État, con la mención "Très Honorable", por su tesis Emilia Pardo Bazán, romancière, dirigida por el Prof. Charles Vincent Aubrun.

En 1975 es nombrada Professeur Titulaire en la Université de Nice, "à titre personnel, par les instances nationales". En los años siguientes desempeña diversos cargos universitarios, tanto locales como nacionales; entre ellos, la Vicepresidencia de la Société des Hispanistes

\footnotetext{
5 Fuente: Hommage à Nelly Clemessy, textes réunis par Gérard, volume préparé par Jean Louis Brau et Gérard Lavergne, Paris: Les Belles Lettres, 1993. Datos actualizados por J.M.G.H. y Cristina Patiño Eirín.
} 
Français de I'Enseignement Supérieur. En 1989 solicita y obtiene el retiro, por razones personales, y es nombrada Professeur Émérite de la Université de Nice.

\section{Publicaciones}

\section{Sobre Emilia Pardo Bazán}

[N. Legal], La Comtesse de Pardo Bazán devant les grandes préoccupations de son temps, D.E.S., Paris, 1954 (Directeur Professeur Aubrun).

[N. Legal], "Emilia Pardo Bazán et Don Perfecto Feijoo", Bulletin des Langues NéoLatines, $\mathrm{n}^{\circ} 162$ (1962), pp. 32-39.

[N. Legal], "Emilia Pardo Bazán poétesse", Bulletin des Langues Néo-latines, n 162 (1962), pp. 40-45.

[N. Legal], "Doña Emilia Pardo Bazán et la Real Academia Española", Annales de la Faculté des Lettres d'Aix-en Provence, CCCVII (1963), pp. 243-262.

[N. Legal], Emilia Pardo Bazán, contes perdus et retrouvés, Thése de Doctorat de 3ème cycle, Montepellier, 1967-68, 268 pp.+ 542 pp. (textes recuellis). (Directeur Professeur Flecknakioska).

[N. Legal], "Contribution à l'étude de Heine en Espagne, Emilia Pardo Bazán, critique et traductrice de Henri Heine", Annales de la Faculté des Lettres de Nice, 3 (1968), pp 73-85.

"En torno a unas cuartillas de Doña Emilia. La Esfinge [fragmento de una novela abandonada]", Revista "José Cornide» de Estudios Coruñeses, "Homenaje a doña Emilia Pardo Bazán", VII, núm. 7 (1971), pp. 7-29.

"El ideario de doña Emilia Pardo Bazán: sus ideas estéticas, sus preocupaciones políticas y sociales", Revista "José Cornide» de Estudios Coruñeses, "Homenaje a doña Emilia Pardo Bazán", VII, núm. 7 (1971), pp. 129-146.

Les Contes d'Emilia Pardo Bazán (Essai de classification), Paris: Centre de Recherches Hispaniques, Institut d'Études Hispaniques, 1972.

Emilia Pardo Bazán, romancière (La critique, la théorie, la pratique), Paris: Centre de Recherches Hispaniques, 1973; traducción al español de Irene Cambra: Emilia Pardo Bazán como novelista. De la teoría a la práctica, Madrid: Fundación Universitaria Española, 1982.

Emilia Pardo Bazán, abogada de Europa en España, Madrid: Fundación Universitaria Española, 1975.

"Emilia Pardo Bazán et l'art du conte", Annales de la Faculté des Lettres et Sciences Humaines de Nice, (septiembre de 1975), pp. 190-197.

"Emilia Pardo Bazán et les littératures étrangéres", Nationalisme et Cosmopolitisme dans les littératures ibériques au XIXème siécle, Centre d'Études Ibériques et Ibéro-Americaines du XIXème siécle, Université de Lille III, 1975, pp. 105-118.

"La Comtesse de Pardo Bazán et le conte rural", Mélanges offerts à Charles Vincent Aubrun, Paris: Éditions Hispaniques, 1975, I, pp. 191-200.

"«El contador», un conte inédit d'Emilia Pardo Bazán", Mélanges en hommage à Jean Villegier, Bulletin des Langues Néo-Latines, n 213 (1975), pp. 59-67.

"D’Emilia Pardo Bazán à Ramón del Valle-Inclàn", TILAS, Revue de I'Institut d'Études Ibériques et Latino-américaines de I'Université de Strasbourg, 1975, pp. 129-139. 
"Emilia Pardo Bazán et le conte fantastique", Mélanges à la mémoire d'André JouclaRuau, Université de Provence, Éditions de l'Université de Provence, 1978, I, pp. 565-575.

"À propos de Los Pazos de Ulloa, de Emilia Pardo Bazán", Centre de Recherches d'Histoire et littérature en Europe au XVIIIème et au XIXème siècles. Université de Besançon, 1979, II, pp. 47-61.

"Une correspondance littéraire: Emilia Pardo Bazán à Narciso Oller", Aspects des Civilisations Ibériques, Amérique Latine, Espagne, Centre Interdisciplinaire d'Étude et de Recherche sur I'Expression Contemporaine, Université de Saint-Étienne, 1979, pp. 169189.

"Le citadin aux champs ou le fausse idylle: Bucólica, de Emilia Pardo Bazán", L'Homme et l'Espace dans la Littérature, les Arts et l'Histoire en Espagne et en Amérique Latine au XIXème siècle, Université de Lille III, 1985, pp. 52-63.

"Persistance de l'esprit anti-napoléonien dans le conte espagnol (2ème moitié du XIXème siecle, début du XXème)", Les Espagnols et Napoléon. Publications du Centre de Recherches d'Études Hispaniques de I'Université d'Aix-en-Provence, 1985, pp. 230-239.

"Arte e idealismo religioso en La Quimera de Emilia Pardo Bazán", Homenaje a José Antonio Maravall, Madrid: C.S.I.C., 1985, vol. 1, pp. 419-429

Edición anotada, con introducción biográfica y crítica, de Los Pazos de Ulloa, Madrid, Espasa Calpe, 1987.

"En torno al realismo de El Cisne de Vilamorta de Emilia Pardo Bazán", Iris, "Aspects du Naturalisme en Espagne", Montpellier, 1988/1, pp. 485-496.

"De La cuestión palpitante a La Tribuna: teoría y praxis de la novela en Emilia Pardo Bazán", en Realismo y Naturalismo en España en la segunda mitad del siglo XIX, Yvan Lissorgues, editor, Barcelona, Anthropos - Université de Toulouse-Le Mirail, 1988, pp. 485-496.

"De Los Pazos de Ulloa a La Madre Naturaleza: Don Julián y el tema del amor prohibido", en Estudios sobre "Los Pazos de Ulloa», Marina Mayoral, coordinadora, Madrid: Cátedra - Ministerio de Cultura, 1989, pp. 51-59.

Prefacio y traducción al francés de Los Pazos de Ulloa: Le Cháteau d'Ulloa, Mayenne, Éditions Viviane Hamy, 1990.

"Selva, un inédit de doña Emilia Pardo Bazán", Homenagem a Eduardo Lourenço: Colectânea de estudos, Lisboa: Instituto de Cultura e Lingua Portuguesa, Ministerio de Educaçao - Nice: Université de Nice, 1992, pp. 145-158.

"Emilia Pardo Bazán", en Historia de la literatura española, dirigida por Jean Canavaggio; edición española de Rosa Navarro Durán, Tomo V, Madrid: Ariel, 1995, pp. 187-192.

"Selva, una tentativa frustrada de novela policíaca", en Estudios sobre Emilia Pardo Bazán. In Memoriam Maurice Hemingway, José Manuel González Herrán, editor, Santiago de Compostela, Universidad - Consorcio de Santiago, 1997, pp. 85-96.

"Unas cartas de Emilia Pardo Bazán a Benito Pérez Galdós" en A Further Range. Studies in Modern Spanish Literature from Caldos to Unamuno. In Memoriam Maurice Hemingway, Anthony H. Clarke, Editor, Exeter: University of Exeter Press, 1999, pp. 136-144. 
"Emilia Pardo Bazán y Perfecto Feijoo: elogio y defensa del folklore musical gallego", La Tribuna. Cadernos de Estudos da Casa Museo Emilia Pardo Bazán, 2 (2004), pp. 65.76. "Emilia Pardo Bazán, novelista", en A. Ma Freire (ed.), Estudios sobre la obra de Emilia Pardo Bazán. Actas de las Jornadas conmemorativas de los 150 años de su nacimiento, A Coruña: Fundación Pedro Barrié de la Maza, 2003, pp. 39-46.

"A propósito de las fuentes históricas de Misterio, novela de Emilia Pardo Bazán", La Tribuna. Cadernos de Estudos da Casa Museo Emilia Pardo Bazán, 4 (2006), pp. 29-42.

\section{Sobre otros temas}

[N. Legal], "Les académiciennes espagnoles du XVIIIIème siècle. Précédents en faveur de I'admision des femmes à la R.A.E.", Mélanges à la mémoire de Jean Sarrailh, Paris: Centre de Recherches de I'Institut d'Études Hispaniques, 1966, pp, 41-45.

L'Espagne de la Restauration (1874-1902), Paris: Col. Bordas-Études, 1973.

"Lázaro, la primera novela de Jacinto Octavio Picón", Cuadernos Hispanoamericanos, no 319 (1977), pp. 37-48.

“Une page d'histoire sociale de l'Espagne. Carmen de Burgos et la polémique sur le divorce", Annales de la Faculté des Lettres de Nice, n 30 (1978), pp. 155-168.

"Roman et féminisme au XIXème siécle. Le thème de la mal mariée chez Jacinto Octavio Picón", Hommage des Hispanistes Français à Noel Salomon, Barcelona: Laia, 1979, pp. 185-198.

"Le nouvel itinéraire espagnol de T'Serstevens: un regard sur le paysage espagnol avant le «boom» touristique des deux dernières décenies", Tourisme et Paysages, Actes du Colloque de Nice, octobre 1982.

"Histoire légendaire et fortune littéraire d'un troubadour galicien: Macías o Namorado", Régards sur le Moyen Age et la Rennaisance, Mélanges Jean Armat, Faculté des Lettres et Sciences humaines de Nice, $n^{\circ} 39$ (1982), pp. 453-463.

"À propos de la réapparition des personages dans l'oeuvre romanesque de Pérez Galdós". Hommage a Louise Bertrand. Annales de la Faculté des Lettres et Sciences humaines de Nice, $\mathrm{n}^{\circ}$ 23, $1^{\mathrm{è}}$ série (1982), pp. 87-97.

"Alfredo Brañas et le régionalisme galicien en 1889", en Nationalisme et littérature en Espagne et en Amérique Latine au XIXème siècle, coord. Cl. Dumas, Université de Lille III, 1982, pp. 50-62.

"Galdós et l'émancipation de la femme espagnole, de La Batalla de Arapiles à Tristana", Publications de la Faculté des Lettres et Sciences humaines de Nice, n² 26, 1è série (1983), pp. 41-51.

"La femme dans la latinité hispanique", Coloque La Latinité d'Aujourd'hui, Les Amis de Sèvres, Paris, 1983.

"Le Naturalisme en Espagne", Cahiers d'Études Romanes, Université de Provence (AixMarseille) $n^{\circ} 8$ (1983), pp. 41-55.

"L'Espagne de Domenico Scarlatti", Domenico Scarlatti, 13 Recherches, Les Cahiers de la Societé de Musique ancienne de Nice (Colloque International de Nice, 1985), nº 1, pp. 16-23. 
"Unas claves posibles de la sensibilidad romántica de Rosalía de Castro en El primer loco", Actas do Congreso Internacional de estudios sobre Rosalía de Castro, Tomo I, Santiago de Compostela: Consello da Cultura Galega - Universidade de Santiago de Compostela, 1986, pp. 523-527.

"Proceso creativo de Celipín Centeno en Marianela", en Actas del Tercer Congreso Internacional de Estudios Galdosianos, Las Palmas de Gran Canaria: Ediciones del Cabildo Insular, 1989, vol. 2 (1990), pp. 31-38. 